\title{
Numerical Calculations of the Pressure Spike in Elastohydrodynamic Lubrication
}

C.H. Venner and W.E. Ten NAPel University of Twente, P.O. Box 217, Enschede, The Netherlands

The application of advanced multilevel techniques to the solution of the EHL line contact problem has resulted in a multilevel solver of complexity $O(n \ln n)$. The reduction in computing time thus obtained enables the use of large numbers of nodes and allows detailed study of the pressure spike. Both occurrence and height of the spike as a function of the governing parameters were studied. The parameter range where a pressure spike can be expected, and the results of spike height calculations, are presented.

KEYworos: pressure spike, EHL, line contact

\section{NOMENCLATURE}

b = Half-width Hertzian contact region.

$E^{\prime} \quad=$ Reduced modulus of elasticity.

$b=\sqrt{\frac{8 \omega R}{\pi E^{\prime \prime}}}$
$=$ Reduced modulu
$\frac{2}{E^{1}}=\frac{1-\nu_{1}^{2}}{E_{1}}+\frac{1-v_{1}^{2}}{E_{1}}$

$E \quad=$ Elasticity modulus (Young's modulus).

$G \quad=$ Material parameter. $G=\alpha E^{\prime}$

$H \quad=$ Dimensionless film thickness.

$H=\frac{h R}{b^{2}}$

$H_{o} \quad=$ Integration constant in dimensionless film thickness equation.

$H_{\min }=$ Dimensionless minimum film thickness (Moes)

$H_{\min }=\frac{h_{\min }}{R}(2 U)^{-1 / 2}$

$h_{\min }=$ Minimum film thickness.

$h \quad=$ Film thickness.

$L \quad=$ Dimensionless material parameter (Moes).

$L=G(2 U)^{1 / 4}$

$M \quad=$ Dimensionless load parameter (Moes).

$M=W(2 U)^{\times 1 / 2}$ 


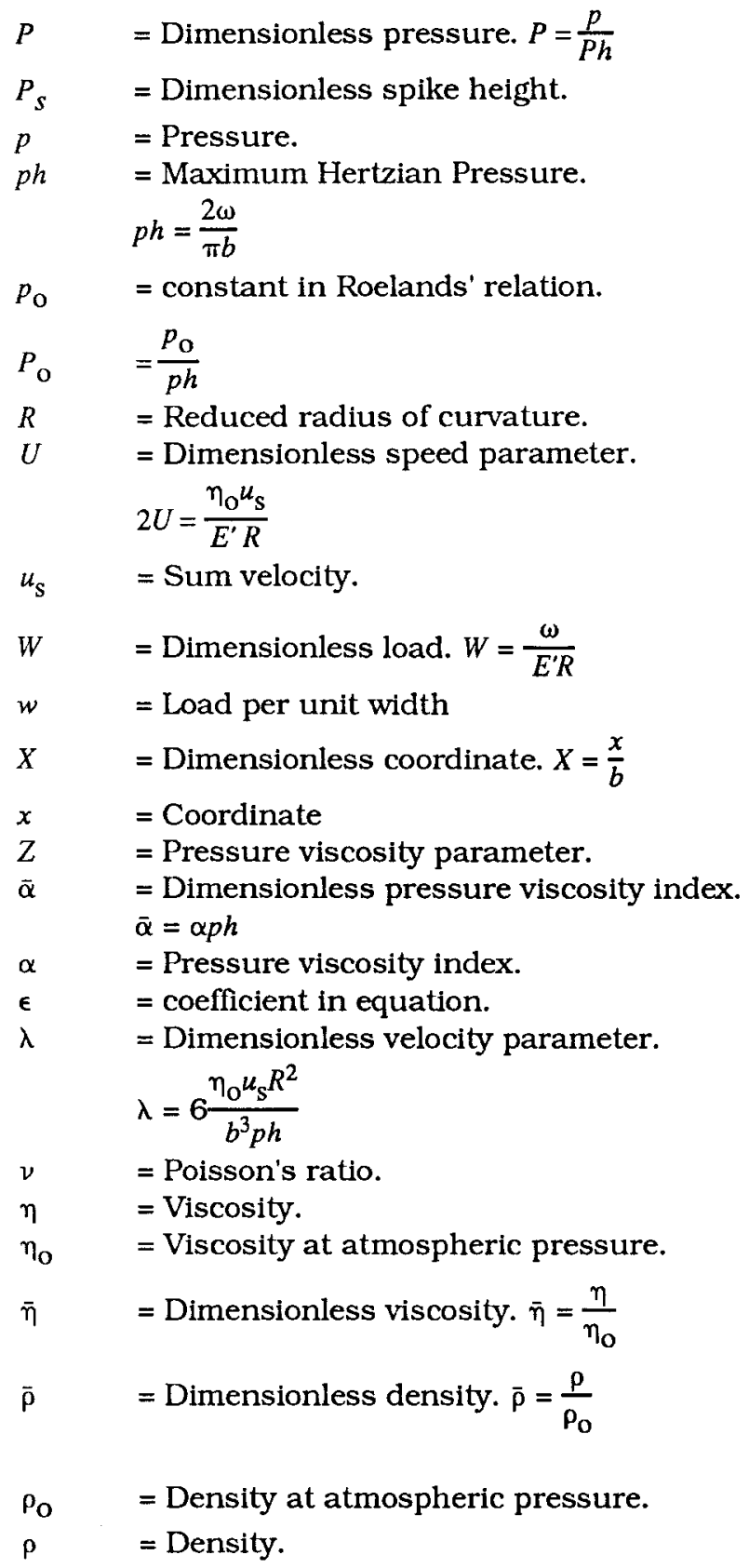




\section{INTRODUCTION}

Over the years. the numerical solution of both the EHL line and point contact problem has received much interest. This has resulted in a number of numerical algorithms of different efficiency for solving these problems. With respect to the line contact problem, the complexity of the presented algorithms varies from $O\left(n^{3}\right)$ (Newton Raphson) to $O(n \ln n)$, if $n$ is the number of grid points used in the calculations, see also reference 1.

One of the most interesting features in the pressure distributions calculated is the so-called 'pressure spike'. The first to report this second local maximum was Petrusevich. ${ }^{2}$ Measurements performed with microtransducers leave no doubt as to the existence of the spike. ${ }^{3.4}$ Its height however has been the subject of many discussions. ${ }^{5,6,7.8,9}$ For example, Kostreva ${ }^{5}$ and Lubrecht ${ }^{7}$ argue that the spike might be a singularity for some specific situations whereas, on the other hand, Hamrock et al ${ }^{6}$ and Pan et $a l^{10}$ present formulas giving the spike height as a function of the governing parameters, ${ }^{6.10}$ and Bisset et a ${ }^{8}$ claim the spike to be finite and smooth provided it is studied at a sufficiently small length scale.

It is commonly accepted that the cause of the spike is the exponential relation between viscosity and pressure. No spike is found if the lubricant was assumed to behave isoviscously. ${ }^{9,11}$ Furthermore, for the same conditions, the spike is higher if the Barus viscosity pressure relation is used instead of the Roelands equation. Similarly, higher spikes are found if the lubricant is assumed to behave incompressibly. ${ }^{9.11}$ The interest in the spike is not just of academic nature, since it may affect surface fatigue and life time in real contact situations. ${ }^{12}$

In this paper the occurrence and the height of the pressure spike in the stationary isothermal EHL line contact situation are investigated. The parameter range where a spike can be expected is presented and the effect of the compressibility, and of the viscosity pressure relation applied, on this parameter range, is shown. Results of spike height calculations using the Roelands equation for both an incompressible and compressible lubricant are presented, and the variation of the spike height with the governing parameters is studied.

\section{EQUATIONS}

All equations are made dimensionless using the Hertzian dry contact parameters. The dimensionless Reynolds equation reads: 
$\frac{d}{d X}\left(\varepsilon \frac{d P}{d X}\right)-\frac{d(\bar{\rho} H)}{d X}=0$

with the cavitation conditions: $P \geq 0$.

$\varepsilon$ is given by:

$\varepsilon=\frac{\bar{\rho} H^{3}}{\bar{\rho} \eta \lambda}$

where

$\lambda=\frac{6 \eta_{\mathrm{o}} u_{\mathrm{s}} R^{2}}{b^{3} P_{h}}$

The dimensionless viscosity $\bar{\eta}$ is pressure dependent. The following relations are used:

$\bar{\eta}=\frac{\eta}{\eta_{0}}=\exp (\ddot{\alpha} P)$

Barus

$\bar{\eta}=\frac{\eta}{\eta_{0}}=\exp \left[\frac{\alpha p_{0}}{z}\left[\left(1+\frac{P}{P_{\mathrm{o}}}\right)^{\mathrm{z}}-1\right]\right]$

Roelands

Where $P_{\mathrm{O}}=p_{\mathrm{O}} / P_{h}$ and $p_{\mathrm{O}}=1.9810^{8}[\mathrm{~Pa}]$

The parameters $\alpha, p_{0}$ and $z$ in the Roelands equation are mutually dependent:

$\alpha=\frac{\ln \left(\eta_{0}\right)+9.67}{p_{0}} z$

In this work $\alpha=1.710^{-8}\left[\mathrm{~Pa}^{-1}\right]$ and $z=0.68$ are used in the calculations with the Roelands equation.

When compressibility is taken into account, the dimensionless lubricant density is assumed to depend on the pressure according to the Dowson and Higginson relation. ${ }^{13}$

The dimensionless film thickness equation reads: 
$H(X)=H_{\mathrm{o}}+\frac{X^{2}}{2}-\frac{1}{\pi} \int \begin{aligned} & \mathrm{X}_{\mathrm{b}} \\ & \mathrm{X}_{\mathrm{a}}\end{aligned} \quad P(Y) \ln |X-Y| d Y$

Finally, the dimensionless force balance equation, stating that the integral over the pressure distribution equals the externally applied load, reads:

$$
\int \begin{aligned}
& \mathrm{X}_{\mathrm{b}} \\
& \mathrm{X}_{\mathrm{a}}
\end{aligned} \quad P(X) d X \frac{\mathrm{p}}{2}
$$

\section{SOLUTION PROCESS}

The equations (1), (2) and (3) are discretized and the resulting discrete equations are simultaneously solved using multilevel, i.e. multigrid techniques. Both the discrete equations and the algorithm are extensively described in reference 1 , and will not be discussed here. Employing this algorithm, the problem can be solved up to the level of truncation errors in $O(n \ln n)$ operations, even for highly loaded situations. This enables solution of the problem with a large number of nodes in reasonable computing times on relatively small computers. For more information on multigrid methods in general the reader is referred to references 14 and 15 .

\section{RESULTS}

All results will be presented in terms of the Moes dimensionless parameters, i.e. load parameter $M$ and material parameter $L$, see reference 16. They are related to the Dowson and Higginson dimensionless parameters $W, U$, and $G$ by:

$M=W(2 U)^{-1 / 2}$

$L=G(2 U)^{1 / 4}$

After 'defining' a spike, the next section presents the parameter range where a spike can be expected depending on the viscosity pressure relation used and whether the lubricant is assumed to behave incompressibly or compressibly. The following section then presents results of spike height calculations. A specific situation is studied in detall and the spike height as a function of $M$ for different values of $L$ is 
presented. The last section compares some results with data presented by other authors.

\section{Onset pressure spike}

With varying $M$ and $L$ different characteristic pressure distributions are generally obtained, see Figure 1. One of the differences between the solutions presented is the number of extremes (locations where $d P / d X=$ $0)$. As a result a parameter for the onset of the pressure spike can be defined as: that value of $L$, given a value of the load parameter $M$, for which the pressure distribution has two extremes and below which only one extreme, located near the centre of the contact, is found. This parameter will be denoted $L_{0}$. For $L>L_{0}$ two local maxima are found, i.e. one in the centre of the contact and another, the spike, near the outlet. As an example, Figure 2 gives details of the pressure distribution in the

Figure 1 Characteristic pressure distributions and film shapes
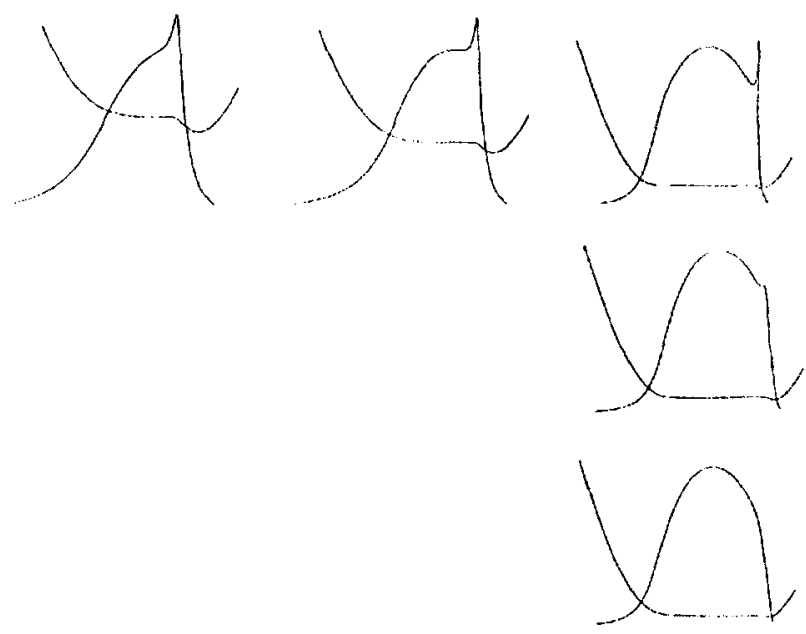
Figure 2 Onset of pressure spike

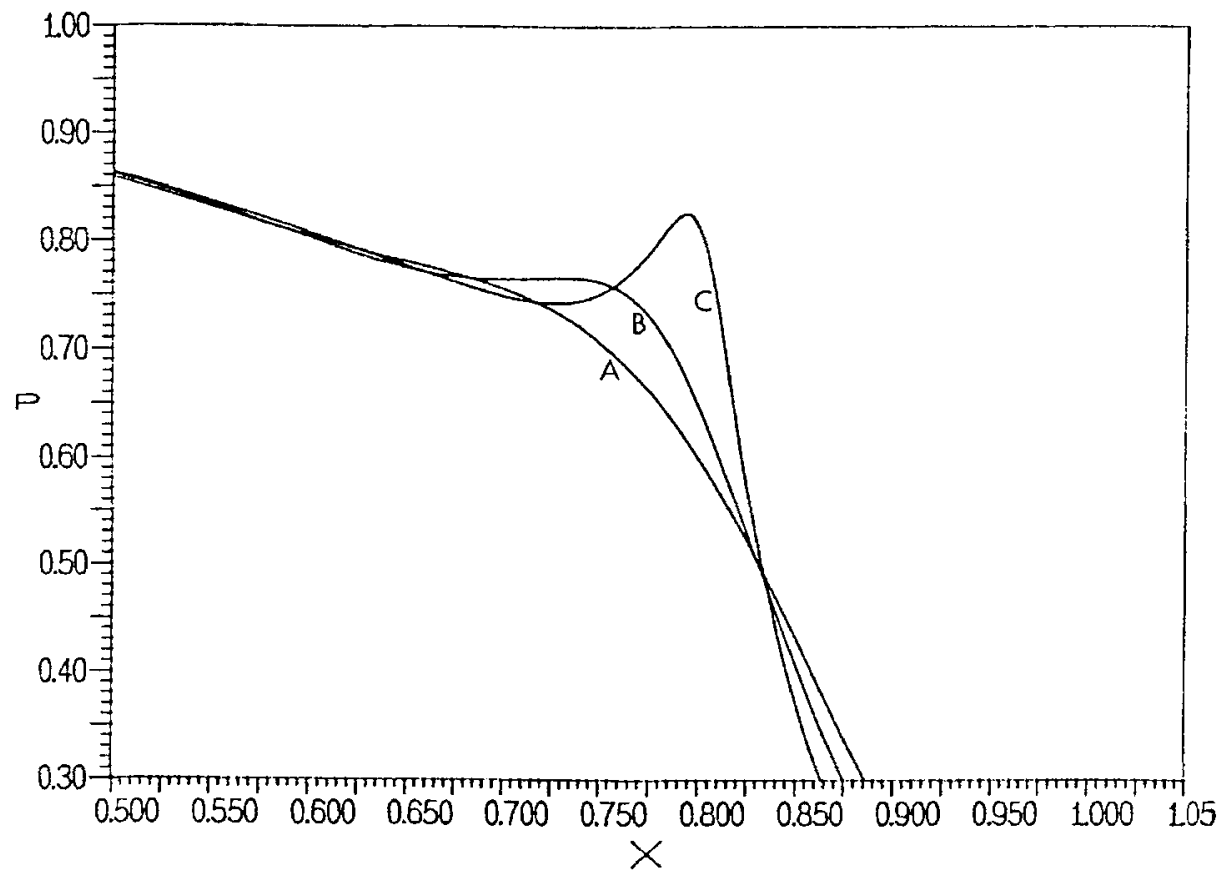

outlet region for a specific value of $M(M \geq 5)$ and three values of $L: L_{A}<L_{0}$, $L_{B} \approx L_{\mathrm{o}}$ and $L_{C}>L_{\mathrm{o}}$. The height of this maximum increases with $L$ and it gradually evolves from a small bump into a spike.

Given some $M$, this onset parameter $L_{0}$ of course depends on the viscosity pressure relation used and differs for a compressible and incompressible lubricant. Figure 3 gives $L_{0}$ as a function of the load parameter $M$ for the different situations of lubricant behaviour, i.e. Barus versus Roelands and incompressible versus compressible. The characteristic pressure distributions prevailing in the regions separated by these curves are also shown. Figure 3 shows that the influence of the lubricant behaviour assumed is rather small. For large values of $M$ the curves run nearly parallel and approximately $L_{\mathrm{o}} \alpha M^{-0.09}$.

From a practical point of view, it may be interesting to plot these curves in the dimensionless film thickness diagram. In that way, it can not only serve to predict the minimum film thickness, but it also provides information on the pressure distribution. See, for example, Figure 4 where the curve for the Roelands/compressible situation is presented in the Moes plot. 
Figure $3 L_{0}$ as function of $M$ and $L$

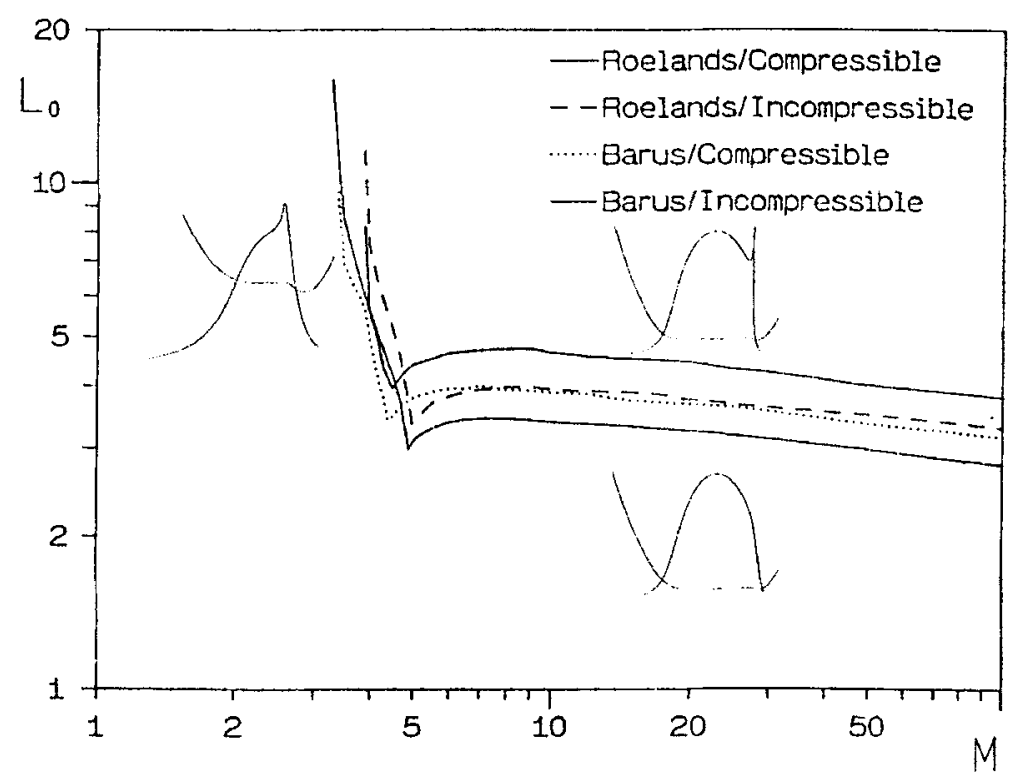

Figure 4 Onset of pressure spike in film thickness plot

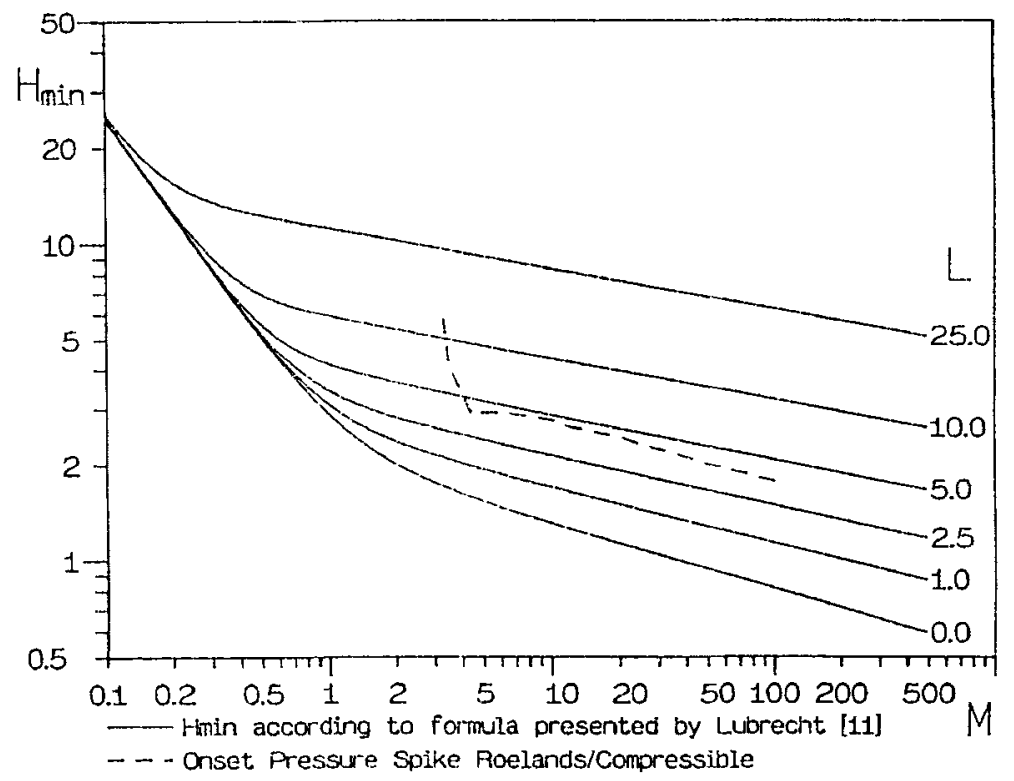




\section{Spike height}

Unless stated otherwise, all results presented in this section have been obtained employing the Roelands equation and assuming a compressible lubricant. Pressure distribution and film thickness for $M$ $=30$ and $L=10$ are presented in Figure 5. Figure 6 shows a detail of the pressure distribution in the spike region for this situation. The calculational domain extended from $X_{a}=-4$ to $X_{b}=1.5$. Convergence to the level of the truncation error was checked as described in reference 11 .

Figure 5 Pressure distribution and film thickness for $M=20$ and $L=10$

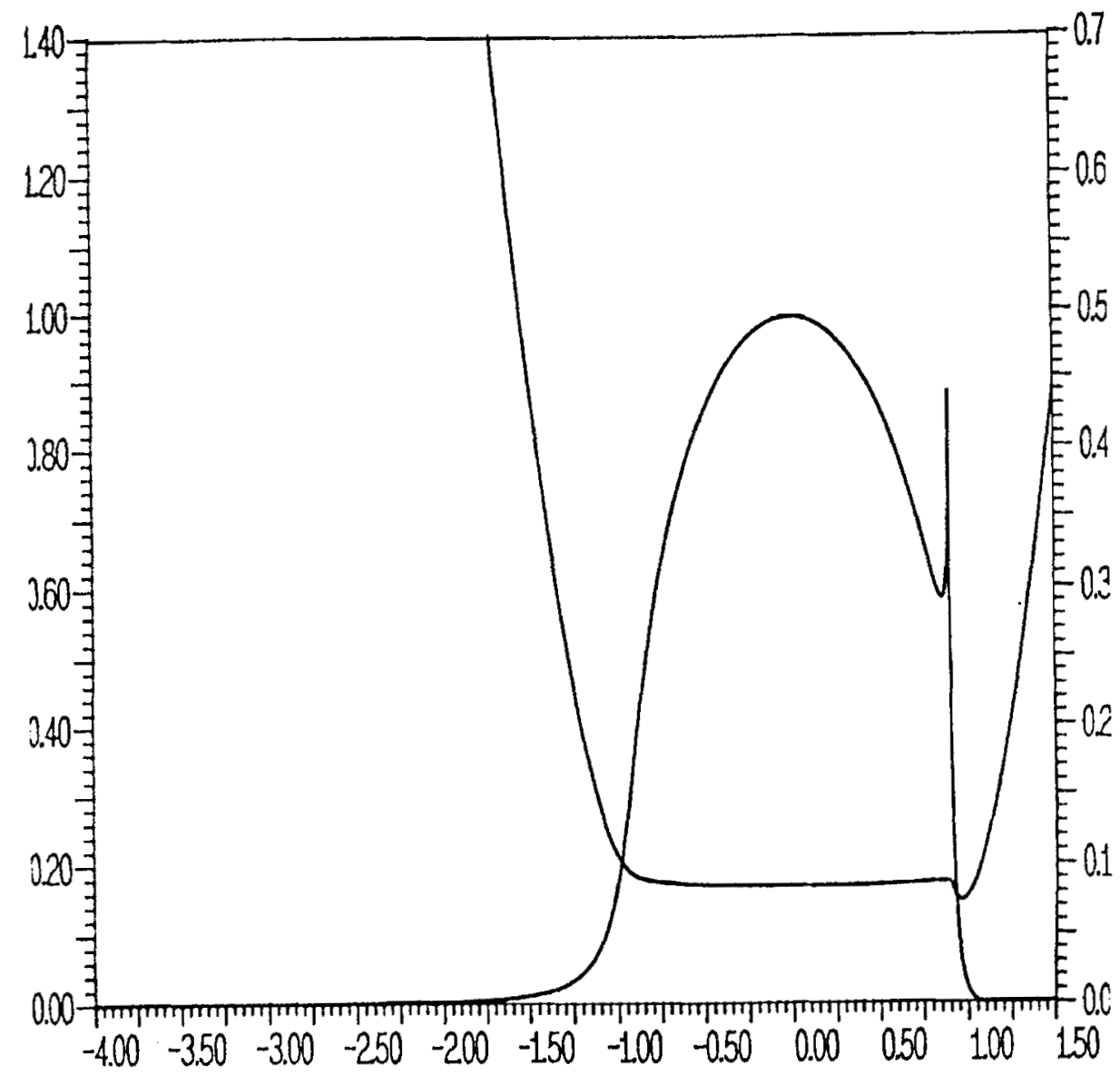


Figure 6 Detail pressure distribution in spike region for $M=20, L=10$

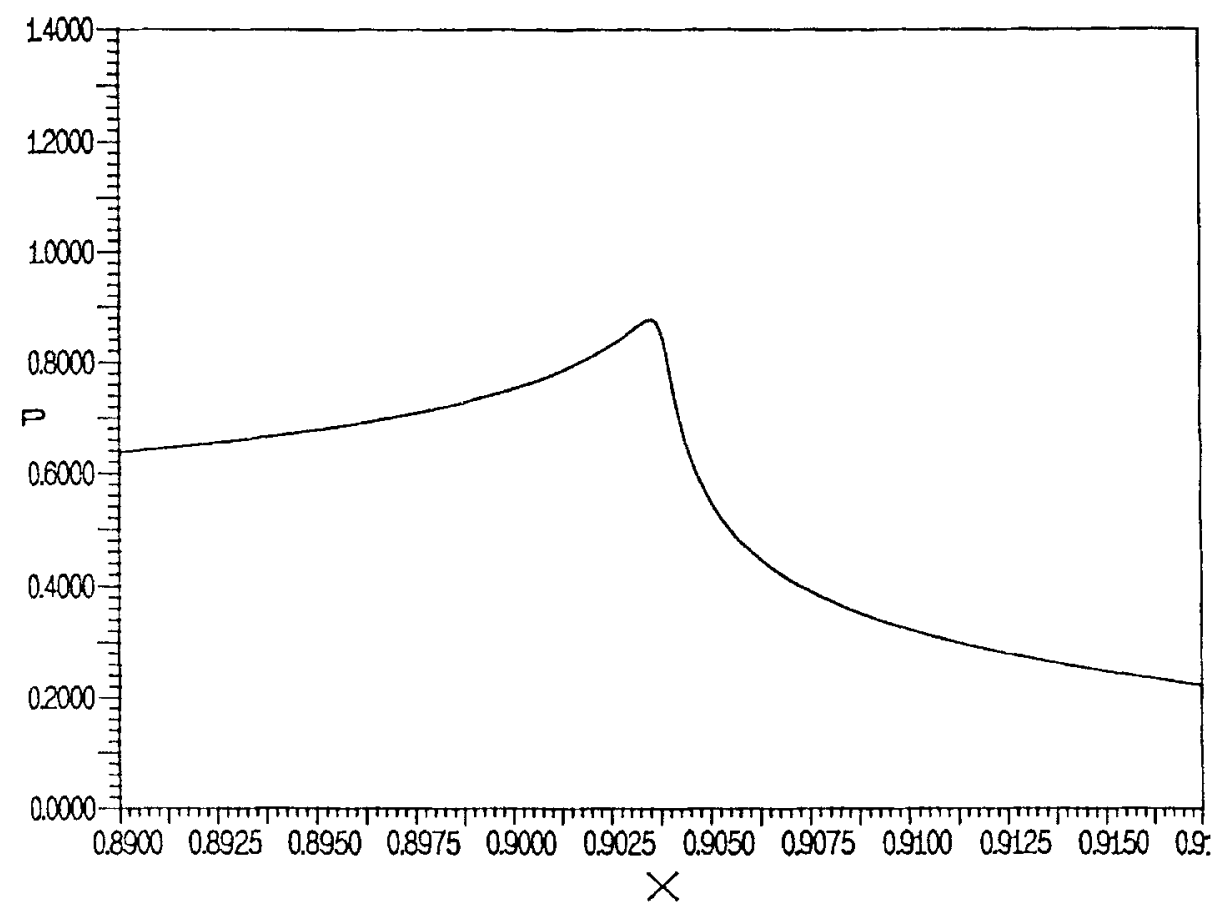

Table 1 gives the dimensionless spike height, $P_{3}$ and the height of the preceding local minimum $P_{m}$ as a function of the number of nodes for this specific situation.

Since a first order upstream discretization was used for the wedge term in equation $1^{1}$ both $P_{3}$ and $P_{m}$ converge first order to a limiting value with successively halving the mesh width. Similarly, pressure distribution and film thickness for other values of $M$ and $L$ where a second local maximum occurs were calculated, and Figure 7 shows the height of this maximum (the spike) as a function of $M$ and $L$. In all situations convergence of the spike with increasing $n$ was checked as described above. Only a limited parameter range is covered. Within the current computing time and computer storage limitations convergence of the spike outside this range could not be shown. To overcome this problem local grid refinement techniques are recommended.

In the parameter range presented, the dimensionless spike height $P_{3}$ decreases with increasing load. This conclusion may not, however, be 
Table 1 Convergence of spike height and preceding minimum as function of the number of nodes

$\begin{array}{cccc}\text { Level } & n & P_{m} & P_{3} \\ 6 & 449 & 0.606 & 0.677 \\ 7 & 897 & 0.590 & 0.710 \\ 8 & 1793 & 0.587 & 0.758 \\ 9 & 3585 & 0.585 & 0.787 \\ 10 & 7169 & 0.584 & 0.825 \\ 11 & 14337 & 0.583 & 0.850 \\ 12 & 28673 & 0.583 & 0.867 \\ 13 & 57345 & 0.583 & 0.875\end{array}$

Figure 7 Dimensionless spike height, Roelands/compressible

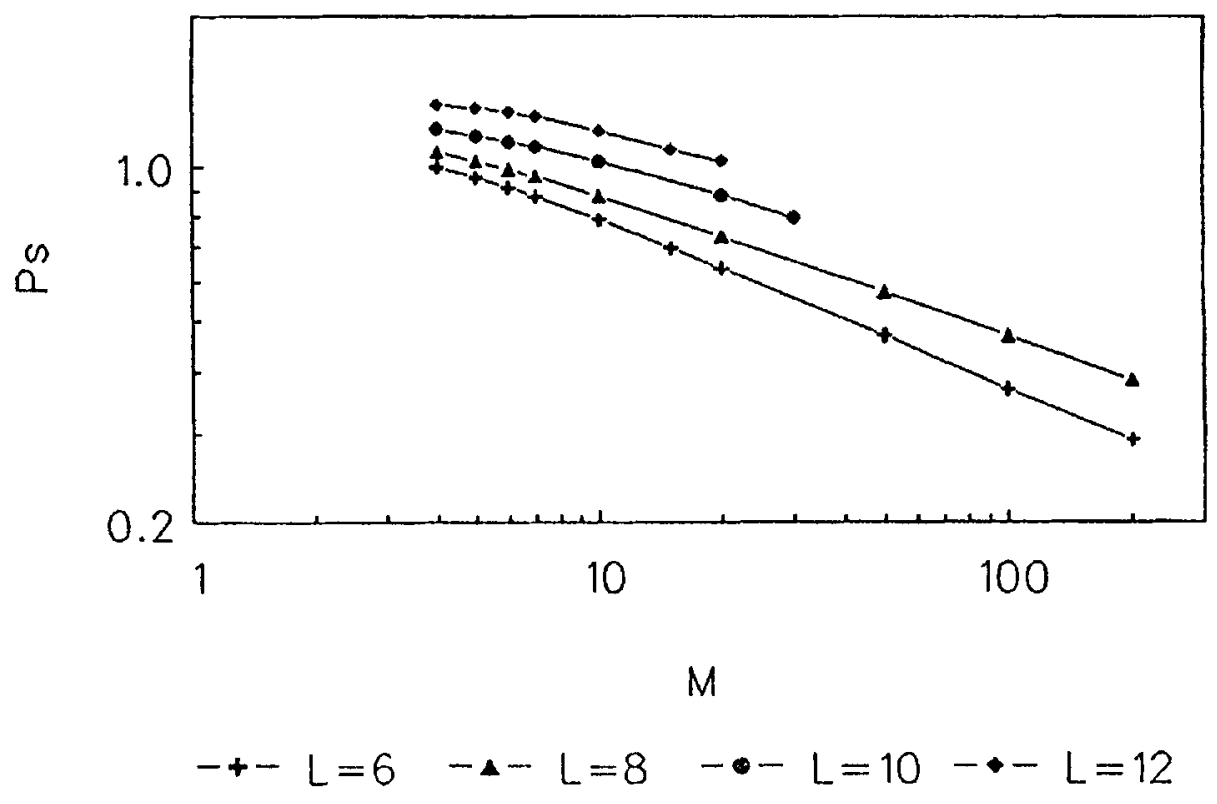


valid over the full parameter range. See, for instance Figure 8, where the spike height results for an incompressible lubricant are presented. In this situation $P_{3}$ decreases with $M$ in a limited range of $M$ and $L$. Beyond this range, $P_{3}$ increases with $M$ for a fixed value of $L$.

\section{Comparison with other authors}

Figure 9 compares some results with data presented by Hamrock et al, ${ }^{6}$ and Pan et $a L^{10}$ In these references some situations with fixed $U$ and $G$ and varying $W$ are presented. In terms of the Moes parameters $L$ is thus fixed whereas $M$ is varied. In all situations, $U=1.010^{-11}$ and $G=5007.2$, thus $L=10.59$. The calculations were performed employing the Roelands relation and assuming a compressible lubricant. Since $L$ is fixed, a curve similar to the ones presented in Figure 7 should be found. For low loads the different results match very well, whereas the differences increases with increasing $M$.

Part of the difference may be accounted for by different values of aand zused in the calculations. However, the rather large deviations of the results presented here and in reference 10 , from the results

\section{Figure 8 Dimensionless spike height, Roelands/incompressible}

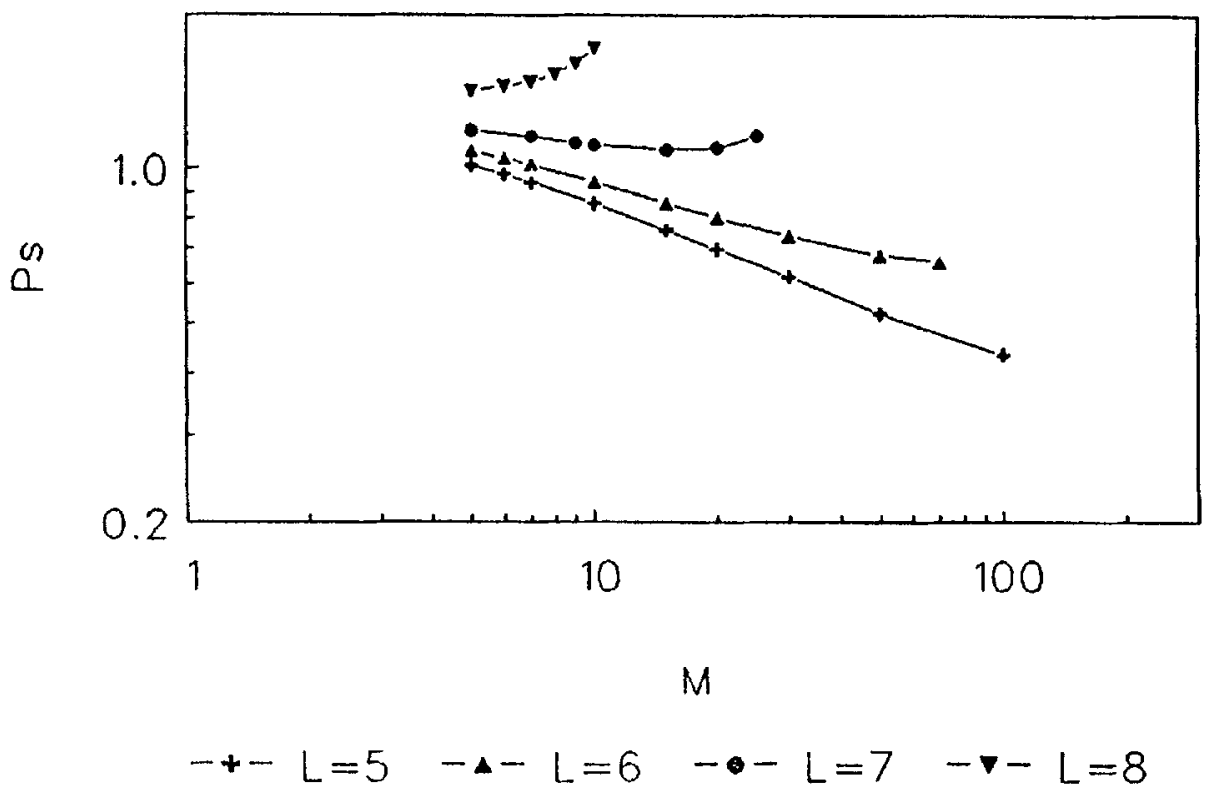


Figure 9 Comparison of spike height results for $L=10.59$

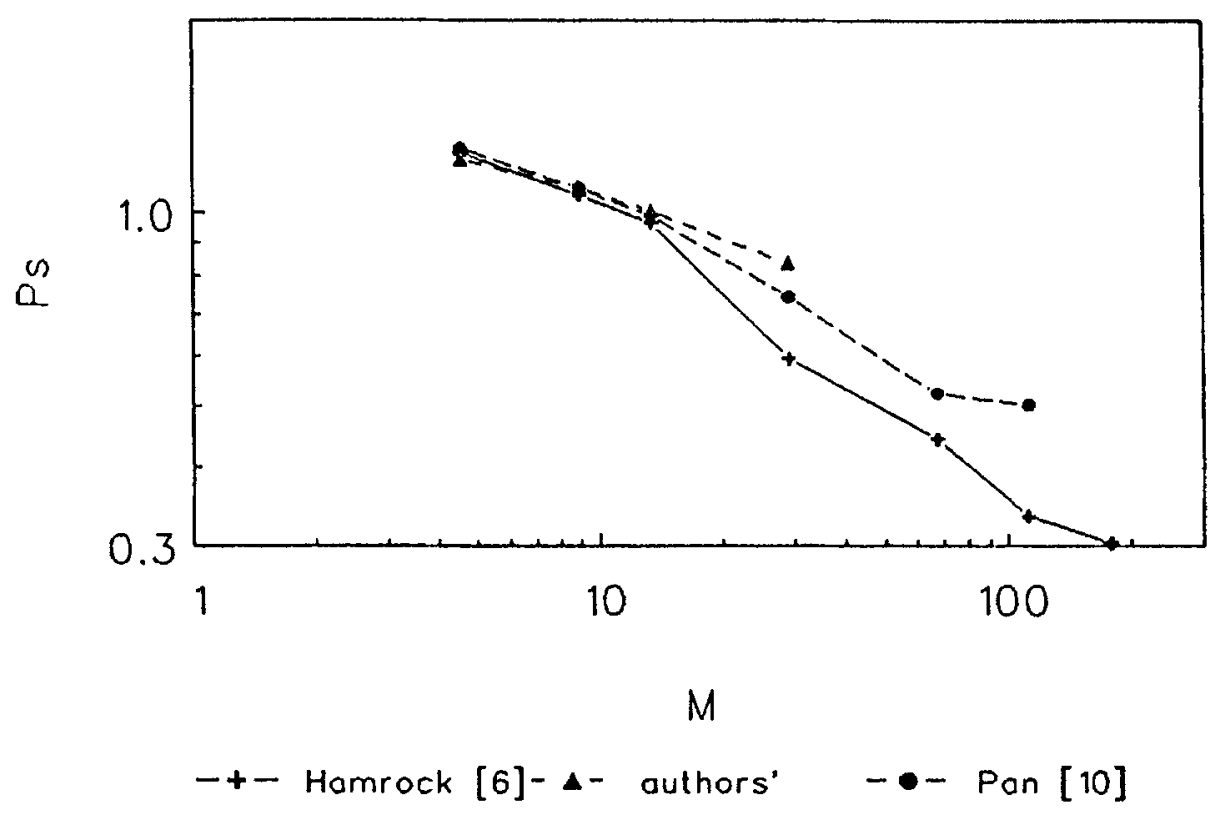

presented in reference 6 , are most likely caused by numerical inaccuracy of the spike height data presented in reference 6 . With increasing $M$ the spike narrows and the number of nodes, needed locally to describe it accurately. increase. If the mesh size in the spike region is not small enough, the spike height will be underestimated.

\section{CONCLUSIONS}

The occurrence and height of the pressure spike as a function of the governing parameters and the pressure viscosity relation used has been studied, both for an imcompressible and compressible lubricant. The parameter range where a second local maximum in the pressure distribution can be expected for the different types of lubricant behaviour, as well as results of spike height calculations, were presented. Although, due to both computing time and computer storage limitations, only part of the parameter range was covered some new insights regarding the variation of the spike height with varying dimensionless load and material number were obtained. With respect to previously presented spike height formula. care should be taken only to 
use within the parameter range of their validity.

\section{ACKNOWLEDGEMENT}

The authors would like to thank prof. A. Brandt and Dr. A.A. Lubrecht for the many helpful discussions with respect to the development of the algorithm. Part of that work was done during a stay at the Weizmann Institute, Rehovot, Israel, sponsored by the faculty of Mechanical Engineering of the University of Twente. The research was also sponsored by SKF Engineering and Research Center B.V., Nieuwegein, The Netherlands.

\section{REFERENCES}

1. Venner, C.H., Ten Napel, W.E., and Bosma, R., 'Advanced Multilevel Solution of the EHL Line Contact Problem', paper presented at the 1989 ASME/STLE Tribology conference, Fort Lauderdale, Florida, USA.

2. Petrusevich, A.I., 'Fundamental Conclusions From the Contact Hydrodynamic Theory of Lubrication', Izv. Akad. Nauk SSR (OTN), 2, 209 1951.

3. Safa, M.M.A., 'Elastohydrodynamic Studies Using Thin Film Transducers', $\mathrm{PhD}$ Thesis, Dept. of Electrical Engineering. Imperial College of Science and Technology, London, 1982.

4. Baumann, H., Von Frey, D., and Haller, R., 'Druck und Temperaturverteilungen in EHD-Kontakten', Tribologie und Schmierungstechnik, 35, 841988.

5. Kostreva, M.M., 'Pressure Stability Considerations in Elasto Hydrodynamic Lubrication Models', ASME JOT, 108, 5511984.

6. Hamrock, B.L., Lee, R.T., and Houpert, L.G., 'Parametric study of performance in elastohydrodynamic lubricated line contacts', Proc. of the 1986 Leeds-Lyon Symposium on Tribology.

7. Lubrecht, A.A., Ten Napel, W.E., and Bosma, R., 'Multigrid, An Alternative Method for Calculating Film Thickness and Pressure Profiles in Elastohydrodynamically Lubricated Line Contacts', ASME JOT, 108, 551 1986.

8. Bisset, E.J., and Glander, D.W., 'A Highly Accurate Approach That Resolves the Pressure Spike of Elastohydrodynamic Lubrication', ASME JOT, 110. 2411988.

9. Hamrock, B.J., Ping Pan, and Rong-Tsong Lee, 'Pressure Spikes in Elastohydrodynamically Lubricated Conjunctions', ASME JOT, 110, 279 1988.

10. Ping Pan, and Hamrock, B.J., 'Simple Formulas for Performance Parameters in Elastohydrodynamically Lubricated Line Contacts', paper presented at the 1988 ASME/STLE Tribology conference, Baltimore. USA. ASME JOT, Preprint 88-Trib.-38.

11. Lubrecht, A.A., The Numerical Solution of the Elastohydrodynamically Lubricated Line- and Point Contact Problem Using Multigrid Techniques', 
PhD Thesis, University of Twente, Enschede, The Netherlands, 1987.

12. Houpert, L., Ioannides, E., Kuypers, J.C. and Tripp, J., The Effect of the EHD Pressure Spike on Rolling Bearing Fatigue', ASMEJOT, 109, 4441987.

13. Dowson, D.J., and Higginson, G.R., Elasto-Hydrodynamic Lubrication, Oxford, Pergamon, lst edition, 1966.

14. Brandt, A., 'Multigrid Techniques: 1984 Guide, With Applications to Fluid Dynamics', Gesellschaft für Mathematik und Datenverarbeitung $\mathrm{MBH}$ Bonn, 1984.

15. Briggs, W.L., A Multigrid Tutorial SLAM, 1988.

16. Moes, H., 'Discussion on a paper by D. Dowson', Proc. Inst. Mech. Engrs. $180,2441965$.

Paper presented at Eurotrib, Helsinki, 1989 